\title{
RARE KAON AND HYPERON DECAYS IN NA48 EXPERIMENT
}

\author{
N.Molokanova $^{a}$ \\ Joint Institute for Nuclear Research, 141980 Dubna, Russia
}

Abstract. Recent results from the experiments NA48/1 and NA48/2 are reported. The first measurement of direct emission and interference terms in $K^{ \pm} \rightarrow \pi^{ \pm} \pi^{0} \gamma$ and the first observation of $K^{ \pm} \rightarrow \pi^{ \pm} e^{+} e^{-} \gamma$ are described. Concerning NA48/1 measurements on radiative hyperon decays are presented.

\section{Introduction}

The series of experiments NA48 have explored many topics in the charged and neutral kaon physics. In this paper we shall discuss some of the most recent measurements produced by two stages of the experimental program: NA48/1 and NA48/2. NA48/1 (2002) has been oriented mainly to the study of rare $K_{s}$ decays and has produced also results in hyperon physics. NA48/2 (2003-2004) was designed to search for direct CP-violation in $K^{ \pm}$decays, but also many other results in rare decays have been achieved.

\section{The radiative decay $K^{ \pm} \rightarrow \pi^{ \pm} \pi^{0} \gamma$}

The decay channel $K^{ \pm} \rightarrow \pi^{ \pm} \pi^{0} \gamma$ is one of the most interesting and important channels for studying the low energy structure of the QCD. Three components contribute to $K^{ \pm} \rightarrow \pi^{ \pm} \pi^{0} \gamma$ decay amplitude: the Inner Bremsstrahlung (IB) associated with the decay $K^{ \pm} \rightarrow \pi^{ \pm} \pi^{0}$ in which the photon is emitted from the outgoing charged pion, Direct Emission (DE) from the vertex and the interference (INT) between these two. The $K^{ \pm} \rightarrow \pi^{ \pm} \pi^{0} \gamma$ decays are described in terms of two kinematic variables: the kinetic energy of charged pion in kaon rest frame $\left(T_{\pi}^{*}\right)$ and invariant variable $W^{2}=\left(P_{K} \cdot P_{\gamma}\right)\left(P_{\pi} \cdot P_{\gamma}\right) /\left(m_{K} m_{\pi}\right)^{2}$, where $P_{K}, P_{\pi}, P_{\gamma}$ are the 4-momenta of the kaon, charged pion and odd gamma, respectively. About $124 \cdot 10^{3}$ events were selected in the range $T_{\pi}^{*}<80 \mathrm{MeV}$ and $0.2<W<0.9$. In the previous measurements a lower cut $T_{\pi}^{*}>55 \mathrm{MeV}$ was introduced in order to suppress $K^{ \pm} \rightarrow \pi^{ \pm} \pi^{0} \pi^{0}$ and $K^{ \pm} \rightarrow \pi^{ \pm} \pi^{0}$ background. In NA48/2 measurement these backgrounds are avoided by application of a special algorithm, which detects overlapping gamma in the detector and due to the limit $\pm 10 \mathrm{MeV}$ on the deviation of reconstructed kaon mass from its nominal value. The upper cut on $T_{\pi}^{*}$ rejects $K^{ \pm} \rightarrow \pi^{ \pm} \pi^{0}$ decays. The background in the selected sample is kept under $10^{-4}$. The probability of the photon mistagging (i.e. choice of wrong odd photon) is estimated to be less than $0.1 \%$.

\footnotetext{
${ }^{a}$ e-mail: Natalia.Molokanova@jinr.ru
} 
The preliminary values for the fractions of DE and INT with respect to IB are

$$
\begin{gathered}
\operatorname{Frac}(D E)=\left(3.35 \pm 0.35_{\text {stat }} \pm 0.25_{\text {syst }}\right) \% \\
\operatorname{Frac}(I N T)=\left(-2.67 \pm 0.81_{\text {stat }} \pm 0.73_{\text {syst }}\right) \% .
\end{gathered}
$$

This is the first measurement of a non vanishing interference term in the $K^{ \pm} \rightarrow$ $\pi^{ \pm} \pi^{0} \gamma$ decay.

\section{$3 \quad$ First observation of the decay $K^{ \pm} \rightarrow \pi^{ \pm} e^{+} e^{-} \gamma$}

NA48/2 experiment observed for the first time the radiative decay $K^{ \pm} \rightarrow$ $\pi^{ \pm} e^{+} e^{-} \gamma$. The signal is selected between $480 \mathrm{MeV} / c^{2}$ and $505 \mathrm{MeV} / c^{2}$ in the invariant $\pi^{ \pm} e^{+} e^{-} \gamma$ mass and requiring the invariant $e^{+} e^{-} \gamma$ mass to be greater that $260 \mathrm{MeV} / c^{2}$. Fig.1 displays the projections of this region on the corresponding axes. The crosses represent data while the filled distribution represent different simulated background contribution. 120 candidates were selected with $7.3 \pm 1.7$ estimated background. The main source of BG is the $K^{ \pm} \rightarrow \pi^{ \pm} \pi_{D}^{0} \gamma$ with a lost $\gamma$.
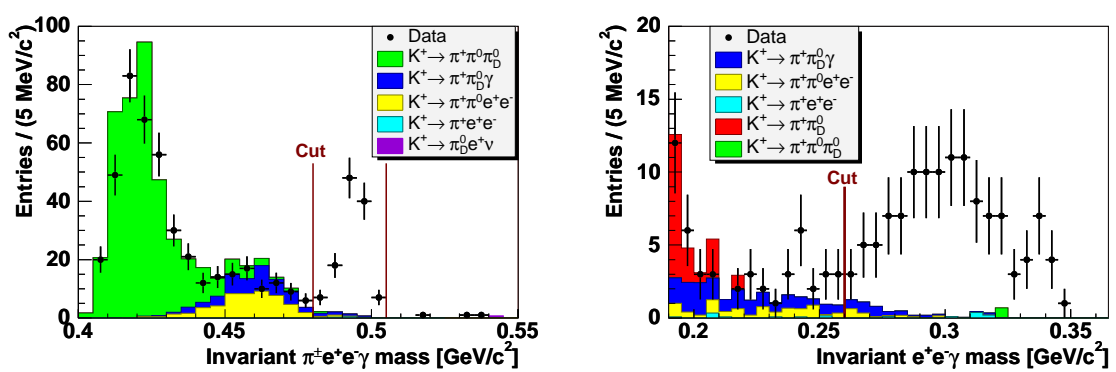

Figure 1: $K^{ \pm} \rightarrow \pi^{ \pm} e^{+} e^{-} \gamma$ decay. The invariant $\pi^{ \pm} e^{+} e^{-} \gamma$ (left) and $e^{+} e^{-} \gamma$ (right) masses with corresponding background distributions. Black crosses represent data distribution.

By using $K^{ \pm} \rightarrow \pi^{ \pm} \pi^{0}$ as normalization channel the branching ratio was preliminary estimated to be

$$
\operatorname{Br}\left(K^{ \pm} \rightarrow \pi^{ \pm} \pi^{0} \gamma\right)=\left(1.19 \pm 0.12_{\text {stat }} \pm 0.04_{\text {syst }}\right) \cdot 10^{-8} .
$$

More details on $K^{ \pm} \rightarrow \pi^{ \pm} e^{+} e^{-} \gamma$ decay analysis could be found in [1].

\section{Weak radiative $\Xi^{0}$ decays}

Up to this day weak radiative hyperon decays as $\Xi^{0} \rightarrow \Lambda \gamma$ and $\Xi^{0} \rightarrow$ $\Sigma \gamma$ are still barely understood. Several theoretical models exist, which give 
very different predictions. An excellent experimental parameter to distinguish between models is the decay asymmetry $\alpha$. It is defined by

$$
\frac{d N}{d \cos \theta}=N_{0}(1+\alpha \cos \theta)
$$

where $\theta$ is the direction of the daughter baryon with respect to the polarization of $\Xi^{0}$ in its rest frame. For example, the decay asymmetry for $\Xi^{0} \rightarrow \Lambda \gamma$ can be measured by looking at the angle between the incoming $\Xi$ and the outgoing proton from the subsequent $\Lambda \rightarrow p \pi^{-}$decay in the $\Lambda$ rest frame. Using this method, the measurement is independent of the unknown initial $\Xi^{0}$ polarization.

The NA48/1 experiment has selected $48314 \Xi^{0} \rightarrow \Lambda \gamma$ and $13068 \Xi^{0} \rightarrow \Sigma \gamma$ candidates (fig.2). The background contributions are $0.8 \%$ for $\Xi^{0} \rightarrow \Lambda \gamma$ and about $3 \%$ for $\Xi^{0} \rightarrow \Sigma \gamma$, respectively.
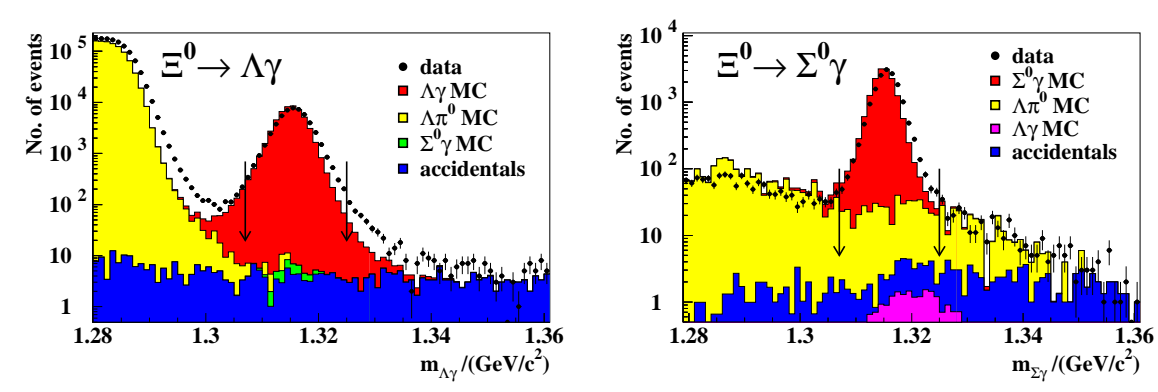

Figure 2: $\Xi^{0} \rightarrow \Lambda \gamma$ (left) and $\Xi^{0} \rightarrow \Sigma \gamma$ (right) signal together with MC expectations for signals and backgrounds.

Using these data, fits to the decay asymmetries have been performed. In case of $\Xi^{0} \rightarrow \Sigma \gamma$, where we have the subsequent decay $\Sigma^{0} \rightarrow \Lambda \gamma$, the product $\cos \theta_{\Xi \rightarrow \Sigma \gamma} \cdot \cos \theta_{\Sigma \rightarrow \Lambda \gamma}$ has to be used for the fit. Both fits show the expected linear behavior on the angular parameters.

After correcting for the well-known asymmetry of $\Lambda \rightarrow p \pi^{-}$, values of

$$
\begin{aligned}
& \alpha_{\Xi^{0} \rightarrow \Lambda \gamma}=-0.684 \pm 0.020_{\text {stat }} \pm 0.061_{\text {syst }} \text { and } \\
& \alpha_{\Xi^{0} \rightarrow \Sigma \gamma}=-0.682 \pm 0.031_{\text {stat }} \pm 0.065_{\text {syst }}
\end{aligned}
$$

are obtained. These values agree with previous measurements by NA48 on $\Xi^{0} \rightarrow \Lambda \gamma[2]$ and $\mathrm{KTeV}$ on $\Xi^{0} \rightarrow \Sigma \gamma$ [3], but are much more precise. In particular the result on $\Xi^{0} \rightarrow \Lambda \gamma$ is of high theoretical interest, as it confirms the large negative value of the decay asymmetry, which is difficult to accommodate for quark and vector meson dominance models. 


\section{$5 \quad$ First observation of $\Xi^{0} \rightarrow \Lambda e^{+} e^{-}$}

In the 2002 run of NA48/1 experiment the weak radiative decay $\Xi^{0} \rightarrow$ $\Lambda e^{+} e^{-}$was detected for the first time [4]. 412 candidates were selected with 15 background events (fig. 3) The obtained branching fraction

$$
\operatorname{Br}\left(\Xi^{0} \rightarrow \Lambda e^{+} e^{-}\right)=\left(7.7 \pm 0.5_{\text {stat }} \pm 0.4_{\text {syst }}\right) \cdot 10^{-6}
$$

is consistent with inner bremsstrahlung-like $e^{+} e^{-}$production mechanism.

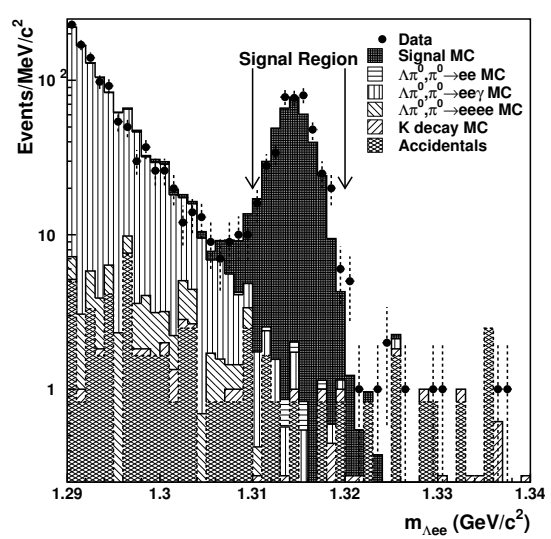

Figure 3: The invariant mass of $\Lambda e^{+} e^{-}$together with the simulated background.

The decay parameter $\alpha_{\Xi \Lambda e e}$ can be measured from the angular distribution

$$
\frac{d N}{d \cos \theta_{p \Xi}}=\frac{N}{2}\left(1-\alpha_{\Xi \Lambda e e} \alpha_{-} \cos \theta_{p \Xi}\right),
$$

where $\cos \theta_{p \Xi}$ is the angle between the proton from $\Lambda \rightarrow p \pi$ decay relative to the $\Xi^{0}$ line of flight in the $\Lambda$ rest frame and $\alpha_{-}$is the asymmetry parameter for the decay $\Lambda \rightarrow p \pi^{-}$. The obtained value

$$
\alpha_{\Xi \Lambda e e}=-0.8 \pm 0.2
$$

is consistent with the latest published value of the decay asymmetry parameter for $\Xi \rightarrow \Lambda \gamma$.

\section{References}

[1] J.R.Batley et al, CERN-PH-EP/2007-033, accepted by Phys.Lett.B.

[2] A.Lai et al, Phys.Lett. B 584, 251 (2004).

[3] A.Alavi-Harati et al, Phys.Rev.Lett. 86 , 3239 (2001).

[4] J.R.Batley et al, Phys.Rev. B 650, 1 (2007). 